\title{
Modulation of Frontostriatal Interaction Aligns with Reduced Primary Reward Processing under Serotonergic Drugs
}

\author{
Birgit Abler, ${ }^{1}$ Georg Grön, ${ }^{1}$ Antonie Hartmann, ${ }^{1}$ Coraline Metzger, ${ }^{2}$ and Martin Walter ${ }^{2,3}$ \\ ${ }^{1}$ Department of Psychiatry, University of Ulm, 89075 Ulm, Germany, ${ }^{2}$ Department of Psychiatry, Otto von Guericke-University, 39106 Magdeburg, \\ Germany, and ${ }^{3}$ Leibnitz Institute for Neurobiology, 39106 Magdeburg, Germany
}

Recently, functional interactions between anteroventral prefrontal cortex and nucleus accumbens (NAcc) have been shown to relate to behavior counteracting reward-desiring (Diekhof and Gruber, 2010). Downregulation of the reward system by serotonin has also been suggested as the mode of action accounting for unsatisfactory effects of serotonin reuptake inhibitors (SSRIs) such as insufficient alleviation or even increase of anhedonia, and loss of interest. However, understanding of the in vivo mechanisms of SSRI-related alteration of the human reward system is still incomplete. Using functional magnetic resonance imaging (fMRI) within a double-blind cross-over within-subjects study design and administering the SSRI paroxetine, the dopamine/norepinephrine reuptake inhibitor bupropione, and placebo for $7 \mathrm{~d}$ each, we investigated a group of 18 healthy male subjects. Under paroxetine, subjects showed significantly decreased activation of the bilateral NAcc during processing of primary rewards (erotic videos), but not under bupropion. Similar to the previous study, analysis of psychophysiological interactions revealed that this downregulation relied on negative interactions between left and right NAcc fMRI signals and the bilateral anteroventral prefrontal cortex that now were significantly enhanced under paroxetine and reduced under bupropion. Individual drug-dependent modulations of interacting brain regions were significantly associated with individual expressions of impulsivity as a personality trait. Our results corroborate and extend previous insights on interregional crosstalk from secondary to primary rewards and demonstrate parallels between active inhibitory control of and serotonergic effects on the dopaminergic reward system's activity.

\section{Introduction}

Modulation of the dopaminergic reward system by serotonin is increasingly attracting attention in systems neuroscience (Seo et al., 2008; Kranz et al., 2010; Hayes and Greenshaw, 2011). A prevalent psychopharmacological example for such an interaction is the reward system's downregulation, which has been suggested to account for unsatisfactory effects of antidepressant serotonin reuptake inhibitors (SSRIs). Symptoms linked to dysfunctional reward processing, such as decreased motivation, loss of interest, and anhedonia, have been observed to be either insufficiently alleviated or even intensified by these drugs (Nutt et al., 2007; Price et al., 2009). Sexual dysfunction, including reduced drive and desire, is a common phenomenon accompanying SSRI intake (Clayton et al., 2002; Serretti and Chiesa, 2009), pointing toward impaired processing of primary rewards. Diminished responsiveness to motivational stimuli under SSRIs has been re-

\footnotetext{
Received Nov. 21, 2011; accepted Dec. 20, 2011.

Author contributions: B.A., A.H., and M.W. designed research; B.A. and A.H. performed research; B.A., G.G., C.M., and M.W. analyzed data; B.A., G.G., and M.W. wrote the paper.

We thank Prof. Dr. C. Hiemke and his staff at the University of Mainz (Germany), Department of Psychiatry and Psychotherapy, for measurements of paroxetine and bupropion blood serum levels.

The authors declare no competing financial interests.

Correspondence should be addressed to Dr. Birgit Abler, Department of Psychiatry, University of Ulm, Leimgrubenweg 12-14, 89075 Ulm, Germany. E-mail: birgit.abler@uni-ulm.de.

DOI:10.1523/JNEUROSCI.5826-11.2012

Copyright $\odot 2012$ the authors $\quad 0270-6474 / 12 / 321329-07 \$ 15.00 / 0$
}

lated to the inhibition of dopaminergic reward circuits by serotonin (Kranz et al., 2010) and is in line with a proposed opponency of dopaminergic and serotonergic effects (Boureau and Dayan, 2011). However, mechanisms remain unclear. Preclinical studies have shown that increased serotonergic tone under SSRIs may facilitate dopaminergic activity under some circumstances, but dopamine release in the nucleus accumbens (NAcc) decreases via stimulation of serotonergic 5HT2C receptors (Alex and Pehek, 2007). Downregulation of reward processing under SSRIs furthermore may relate to reciprocal interactions of prefrontal areas with the NAcc (Carr and Sesack, 2000; Goto and Grace, 2008).

In a recent functional magnetic resonance imaging (fMRI) study in healthy subjects using primary rewards, we demonstrated decreased subcortical reward system activation under steady-state conditions of the SSRI paroxetine but not under the dopamine-norepinephrine reuptake inhibitor bupropion (Abler et al., 2011). No interaction effect of task and medication was observed in prefrontal brain areas. Thus, the design permits investigating how cortico-subcortical functional interactions of reward-related brain areas may modulate the downregulation of reward functions under SSRIs. Similar to a previous report (Diekhof and Gruber, 2010), this issue was investigated using the framework of psychophysiological interaction (PPI) analyses to study whether or how these interactions may be differentially modulated by the different drugs. Diekhof and Gruber (2010) 
have shown an increased negative interaction between the bilateral anteroventral prefrontal cortex (avPFC) and decreased reward-related NAcc activation. This happened whenever subjects successfully inhibited impulses toward gathering a small immediate reward for the sake of a greater future monetary reward.

Accordingly, we hypothesized that the same negative interaction between reward-processing areas and orbitofrontal regions can be observed with primary rewards (erotic stimuli), and that this interaction would be enhanced by the SSRI but not by bupropion. Due to actual knowledge about the serotononergic projections from midbrain's raphé nuclei, we extended the hypothesis derived from the previous study and also investigated the interaction between the raphé nuclei and the NAcc. Again, we expected a negative interaction between these regions, proposing a potential mechanism for the enhanced downregulation of NAcc activity under the influence of SSRIs. Finally, we measured individual expressions of selected personality traits that may predict individual pharmacological effects on the proposed connectivity.

\section{Materials and Methods}

Subjects. Eighteen healthy male, heterosexual subjects aged 23-34 years (mean 25.4 years, SD 2.9 years, three left-handed) took placebo, bupropion, and paroxetine at routine clinical doses for $7 \mathrm{~d}$ each in a counterbalanced order and were included in the fMRI study. The study was approved by the local ethical review board of the University of Ulm. All volunteers gave written informed consent before the study in accordance with the Declaration of Helsinki. Exclusion criteria were any psychiatric, neurological, and/or major medical diagnoses currently or in the past; any serious general medical condition; use of illegal drugs; excessive consumption of caffeine or alcohol; any regular systemic medication; and/or any other medication within the week before the study. Detailed sample characteristics and exclusion criteria were reported previously (Abler et al., 2011). Upon recruitment, subjects completed a German version (Reinecke et al., 2006) of the Massachusetts General Hospital Sexual Function Questionnaire (Labbate and Lare, 2001).

Study design and procedures. The study was set up as a randomized, double-blind, placebo-controlled within-subject cross-over design. Each subject underwent an fMRI scan on three different occasions, once under placebo (gelatin capsule filled with mannitol powder), once under paroxetine (20 mg, capsule with pulverized paroxetine), and once under bupropion (150 mg, capsule with bupropion). All capsules appeared identical. To allow for washout time, scans were separated by intervals of at least $14 \mathrm{~d}$. For each treatment, subjects were instructed to take one capsule each morning for $7 \mathrm{~d}$ to reach steady-state blood levels. On the seventh day, subjects were scheduled for an fMRI scan $2 \mathrm{~h}$ after intake of the last capsule. Blood samples were taken after each fMRI session to assess treatment adherence to bupropion and paroxetine. Blood level analyses after completion of the whole study revealed mean bupropion levels of $59.9 \mathrm{ng} / \mathrm{ml}$ (SD 27.7) and mean paroxetine levels of $28.1 \mathrm{ng} / \mathrm{ml}$ (SD 17.9).

Task and stimuli. During fMRI scans, erotic video clips were presented as primary rewards, alternating with neutral video clips in a standard block design with prolonged visual stimulation. Erotic video clips demonstrated sexual interactions between one man and one or two women (petting, oral sex, and vaginal intercourse) extracted from commercial adult films. The neutral video clips depicted men and women in emotionally neutral, nonerotic interactions (at a shop, at the airport). Nine different video clips of each type were presented for $20 \mathrm{~s}$ each, separated by a $20 \mathrm{~s}$ interstimulus interval with a white fixation cross on a black screen. Video clips were presented in a pseudorandomized order with not more than two consecutive clips of the same type.

fMRI acquisition. A 3.0 tesla Magnetom Allegra scanner (Siemens) equipped with a head coil was used to acquire T1 anatomical volume images $(1 \times 1 \times 1 \mathrm{~mm}$ voxels $)$ and functional magnetic resonance images. Twenty-three transversal slices were acquired with an image size of $64 \times 64$ pixels and a field of view of $192 \mathrm{~mm}$. Slice thickness was $3 \mathrm{~mm}$ with $0.75 \mathrm{~mm}$ gap, resulting in a voxel size of $3 \times 3 \times 3.75 \mathrm{~mm}$. Images were centered on basal structures of the brain, including subcortical regions of interest (basal ganglia and prefrontal regions). Functional images were recorded using a $\mathrm{T} 2{ }^{\star}$-sensitive gradient echo sequence measuring changes in blood oxygenation level-dependent (BOLD) contrast. During viewing of video clips, 487 volumes were obtained at a TR of 1500 ms (TE, $35 \mathrm{~ms}$; flip angle, $90^{\circ}$ ).

$f M R I$ analysis. Image processing and statistical analyses were performed using Statistical Parametric Mapping (SPM5, Wellcome Trust Centre for Neuroimaging, London, UK) with a random effects model for group analyses. Preprocessing of the individual functional scans included realignment to correct for motion artifacts, slice timing, spatial normalization to a standard template [Montreal Neurological Institute (MNI) voxel size after normalization: $2 \times 2 \times 2 \mathrm{~mm}$ ], and smoothing with an 8 mm FWHM Gaussian kernel. Intrinsic autocorrelations were accounted for by an AR(1) model and low-frequency drifts were removed via highpass filtering.

After preprocessing, first-level analyses were performed for each subject. According to the general linear model, we defined two separate regressors to estimate the neural activation associated with the two types of video stimuli (erotic and nonerotic, each against fixation baseline), and the contrast thereof. Video blocks were modeled as timely extended events of $20 \mathrm{~s}$ and convolved with the hemodynamic response function. The six realignment parameters modeling residual motion were also included in the individual models. We defined two regions-of-interest (ROIs) for the left and right NAcc using the masks provided by the Harvard-Oxford cortical and subcortical structural atlases (www.cma. mgh.harvard.edu/fsl_atlas.html). The left-sided ROI consisted of 74 voxels with a voxel size of $2 \times 2 \times 2 \mathrm{~mm}$; the right-sided ROI consisted of 63 voxels.

From each subject, the estimated mean fMRI signal averaged across voxels of each ROI was extracted and served as individual parameter estimates of modeled effects for each condition of erotic and nonerotic video clips against fixation baseline blocks. Main and interaction effects of the factors condition (erotic, nonerotic) and medication (placebo, paroxetine, bupropion) on averaged parameter estimates were tested using a $2 \times 3$ ANOVA. The main and interaction effects were calculated with STATISTICA 6.0 and thresholded at $p<0.05$ to infer significant effects. For the planned post hoc paired $t$ tests computed to test significant treatment effects on the differential fMRI signal (erotic minus nonerotic), we applied a rough false-discovery rate correction to account for the six post hoc tests of interest (adjusted $p=0.029$ ).

PPIs (Friston et al., 1997) were calculated to assess the hypothesized negative functional interactions of the NAcc activation with the bilateral avPFC and raphé nuclei to investigate mechanisms of decreased reward system reactivity under the SSRI. Therefore, the two NAcc ROIs were chosen as seed areas. BOLD signal time series were extracted from these ROIs using the VOI time series extraction utility provided by SPM. The extracted signal time series served as physiological regressors. The main effect of condition (erotic minus nonerotic) was defined as the psychological regressor. The PPI term was built separately for the left and right NAcc, representing the regressors of interest, and were computed using the PPI toolbox of the SPM software. First-level analysis was performed separately for each NAcc ROI and treatment, estimating the variance of voxels according to a general linear model, to determine task-dependent changes of connectivity beyond and above the main effects of task and activation.

Each of these designs yielded one PPI contrast image; that is, six PPI contrast images per subject ( 2 NAcc ROIs $\times 3$ treatment levels). These images were then propagated to two separate random effects analyses (one for each NAcc ROI) to average PPI analyses across subjects. Inference of significant results was restricted to predefined ROIs within the anterovental prefrontal cortex (avPFC, see below) and the periaqueductal gray comprising the anatomical localization of the raphé nuclei (see below). Statistical results of PPI analyses are reported down to the lenient threshold of $p<0.01$ uncorrected, although most of the main and differential effects reported survived corrections for multiple comparisons (these effects are marked in Tables 1-3). We chose the more lenient threshold because we wanted to ascertain whether functional interac- 
tions of the NAcc and avPFC were indeed lateralized or simply weaker in one hemisphere.

For the avPFC ROI, we used the peak voxel of an already statistically inferred negative interaction between the NAcc and the left avPFC (MNI coordinates: $x / y / z=-28 / 56 / 4$ ) from the previous study by Diekhof and Gruber (2010) as a starting point, and defined a $10 \mathrm{~mm}$ sphere around this maximum for the left avPFC ROI. The resulting ROI, comprising an orbitofrontal brain region within Brodmann area 10, was mirrored to establish the avPFC ROI in the right hemisphere $(10 \mathrm{~mm}$ sphere around $x / y / z=28 / 56 / 4)$. The raphé nuclei ROI was defined as a $10 \mathrm{~mm}$ sphere centered on an activation maximum (MNI coordinates: $x / y / z=3 /-30 /$ -18) from a previous study on perception of sexual stimuli (Walter et al., 2007).

To further test the behavioral impact of potentially drug-modulated frontostriatal interactions, correlations between differential (verum vs placebo) PPI effects and individual scores on the Thrill and Adventure Seeking (TAS) subscale of the German version of the Sensation Seeking Scales, Form V (SSS-V) (Beauducel et al., 2003) were calculated. The TAS was chosen first because our previous findings demonstrated correlations between the TAS subscale and brain activity related to monetary rewards (Abler et al., 2006). Second, although slightly different in item construction, the TAS subscale has a close relationship to the Novelty Seeking scale of the Cloninger Temperament and Character Inventory, which was assessed in a previous study on NAcc-avPFC interactions (Diekhof and Gruber, 2010). Correlations of individual estimated heights of interaction effects between NAcc and avPFC, as well as NAcc and raphé nuclei with individual scores of the TAS scale, were calculated using SPM, again restricting the analyses to the voxels within predefined ROIs.

\section{Results}

Subjects reached average sum scores on the SSS-V scale (score, 23.7; SD , 5.7). On three of the four subscales, the present sample's means of scores were within half a standard deviation of the corresponding scores of a reference sample (Beauducel et al., 2003). Scores for the fourth subscale, TAS, were slightly higher (mean, 8.4 points; SD, 2.4) compared with the average of the reference sample.

\section{Effect of erotic stimulation $\times$ treatment interaction in bilateral nucleus accumbens}

As hypothesized, the $2 \times 3$ ANOVA on the NAcc ROI-averaged fMRI signal revealed a significant main effect of condition (erotic vs nonerotic video clips) in left $\left(F_{(2,34)}=22.17, p=0.0002\right)$ and right $\left(F_{(2,34)}=20.08, p=0.0003\right)$ NAcc, and a significant interaction effect with the factor treatment (left NAcc: $F_{(2,34)}=4.93, p=0.01$; right NAcc: $\left.F_{(2,34)}=5.45, p=0.009\right)$. Planned post hoc paired $t$ tests on the difference fMRI signal (erotic minus nonerotic) confirmed a significantly decreased signal magnitude bilaterally when comparing paroxetine to bupropion (left: $t_{(17)}=3.06, p=0.007$; right: $t_{(17)}=$ $3.29, p=0.002)$, and on the right hemisphere when comparing paroxetine against placebo (right: $t_{(17)}=2.05, p=0.028$ ). For the left hemisphere, the contrast paroxetine versus placebo failed significance according to the adjusted $p$ value $\left(t_{(17)}=1.88, p=0.039\right)$. There was a trend toward increased activation under bupropion compared with placebo (left: $t_{(17)}=1.41, p=0.088$; right: $t_{(17)}=$ $1.39, p=0.090)$. No significant correlations were found between differential effects in brain activation and data from questionnaires. Results are depicted in Figure $1 A$.

\section{PPI results}

Parallel to previous findings (Diekhof and Gruber, 2010), we found significant negative psychophysiological interactions between NAcc and avPFC (Table 1) already under placebo, which were markedly pronounced under paroxetine. Interactions be-
A

left Nacc ROI activation: erotic > neutral video clips

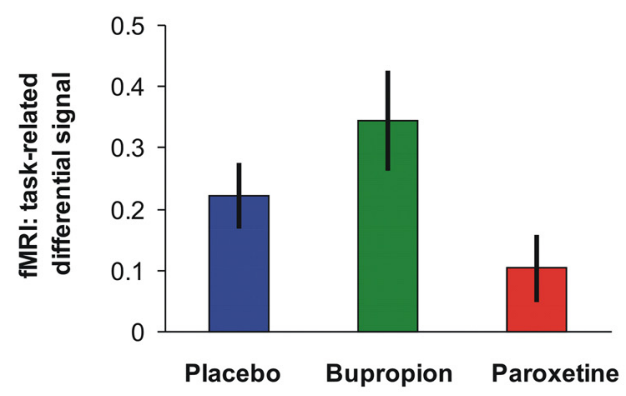

B

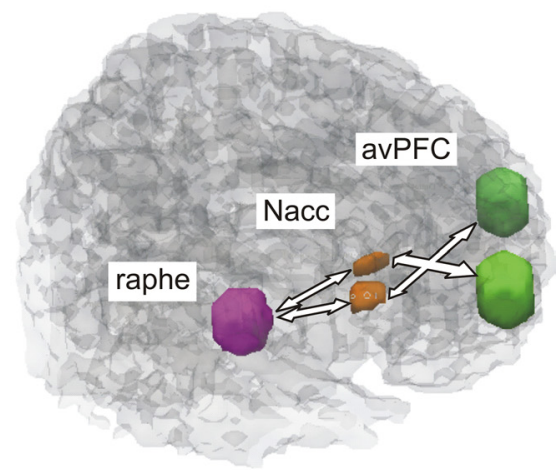

C

PPI: left Nacc and right avPFC

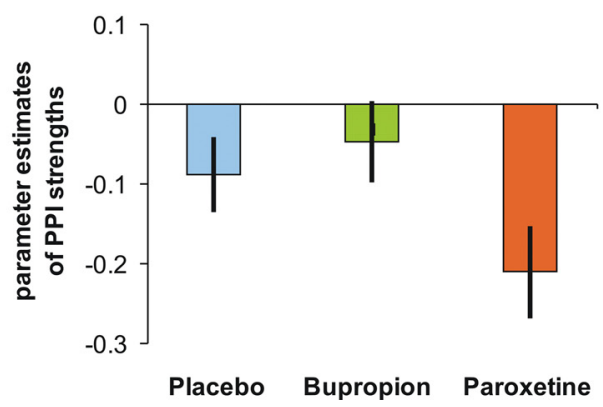

Figure 1. A, Task-related fMRI activation (differences in parameter estimates of modeled effects) within the left NAcc ROI for the contrast erotic minus nonerotic video clips with significantly reduced activation under the selective serotonin reuptake inhibitor paroxetine compared with the dopamine/norepinephrine reuptake inhibitor bupropion and placebo. $\boldsymbol{B}$, ROls for NAcc, defined according to the Harvard-0xford cortical and subcortical structural atlases, for the avPFC defined according to Diekhof and Gruber (2010) and for the raphé nuclei defined according to Walter et al. (2007). C, Parameter estimates of negative PPI strengths between left $\mathrm{NAcc}$ and right avPFC with significantly greater negative interaction under paroxetine than under placebo and bupropion and less negative interaction under bupropion compared with placebo.

tween NAcc and raphé nuclei were significant only under paroxetine. Analyses of treatment-related changes in connectivity of bilateral NAcc with avPFC and raphé nuclei confirmed the prominent effect under paroxetine (Table 2; Fig. 1C): Negative interactions were significantly stronger under paroxetine than under placebo and bupropion. Negative interactions were still stronger under placebo than under bupropion (Table 2; Fig. 1C). No differences were observed for the inverted contrasts of bupropion minus placebo or placebo minus paroxetine.

To further assess the specificity of our preselected ROIs, we reran the group analysis on averaged PPIs under paroxetine anatomically unrestricted over the whole brain $(p<0.001$, uncorrected, with a spatial extent of at least 10 contiguously significant voxels per clus- 
Table 1. Psychophysiological interaction results: negative interactions of left and right NAcc activity with left and right avPFC ROIs and with the raphé nuclei ROI

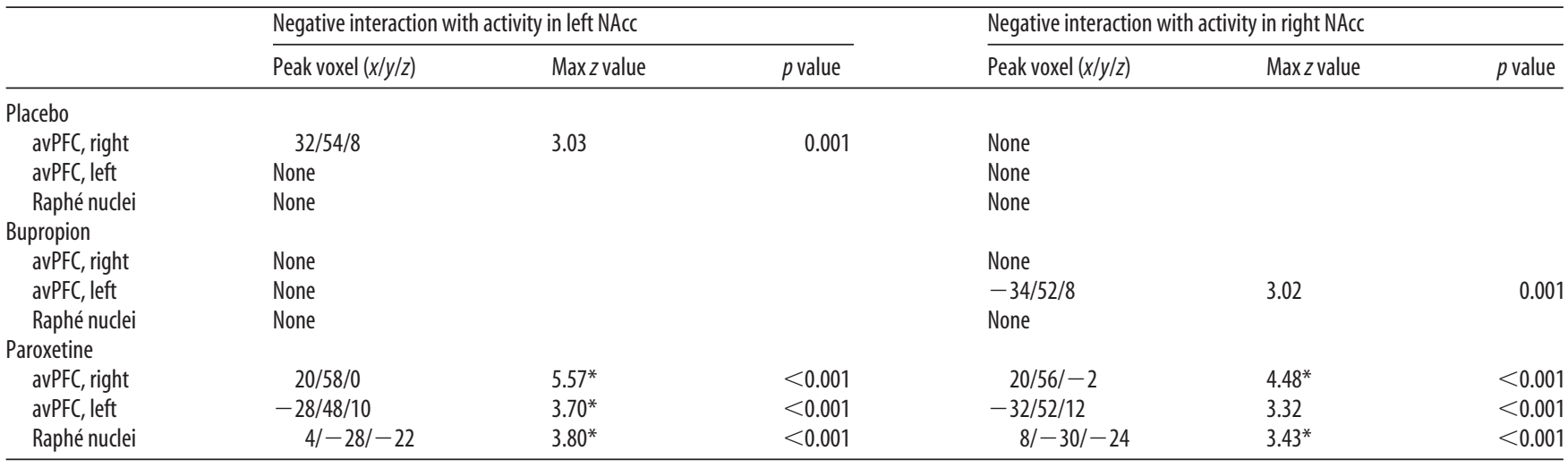

Results reported down to a threshold of voxel-level: $p<0.01$, uncorrected.

*Results survive FWE correction at $p<0.05$.

Table 2. Psychophysiological interaction results: differential interaction expressed in left and right avPFC ROIs and in the raphé nuclei ROI between activity within left and right NAcc and experimental condition (erotic vs neutral video clips)

\begin{tabular}{|c|c|c|c|c|c|c|}
\hline & \multicolumn{3}{|c|}{ Differential interaction with activity in left NAcc } & \multicolumn{3}{|c|}{ Differential interaction with activity in right NAcc } \\
\hline & Peak voxel $(x / y / z)$ & Max $z$ value & $p$ value & Peak voxel $(x / y / z)$ & Max $z$ value & $p$ value \\
\hline \multicolumn{7}{|l|}{ PAR $>$ PLAC } \\
\hline avPFC, right & $22 / 58 / 2$ & $3.36^{*}$ & $<0.001$ & $26 / 56 / 4$ & 2.59 & 0.005 \\
\hline avPFC, left & None & & & $-26 / 50 / 8$ & 2.48 & 0.007 \\
\hline PAG/raphe & $6 /-28 /-22$ & $3.51^{*}$ & $<0.001$ & $6 /-26 /-22$ & 3.16 & 0.001 \\
\hline \multicolumn{7}{|l|}{ PAR $>$ BUP } \\
\hline avPFC, right & $20 / 58 / 0$ & $4.31^{*}$ & $<0.001$ & $28 / 54 / 6$ & $3.32^{*}$ & $<0.001$ \\
\hline avPFC, left & None & & & None & & \\
\hline PAG/raphe & $2 /-28 /-20$ & $4.03^{*}$ & $<0.001$ & $6 /-34 /-20$ & $3.63^{*}$ & $<0.001$ \\
\hline \multicolumn{7}{|l|}{ PLAC $>$ BUP } \\
\hline avPFC, right & $30 / 52 / 8$ & 2.87 & 0.002 & None & & \\
\hline avPFC, left & None & & & None & & \\
\hline PAG/raphe & $-4 /-28 /-18$ & 2.81 & 0.002 & $0 /-34 /-18$ & 3.08 & 0.001 \\
\hline
\end{tabular}

Threshold at voxel-level: $p<0.01$, uncorrected.

*Results survive FDR correction at $p<0.05$.

PAR, paroxetine; PLAC, placebo; BUP, bupropion; PAG, periaqueductal gray.

ter). Results of this analysis are depicted in Figure 2, demonstrating a high anatomical correspondence between results of this analysis and the preselected ROIs.

\section{Correlational findings of PPI and individual TAS scores}

Enhanced negative connectivity between NAcc and avPFC under paroxetine versus placebo was significantly related to higher individual TAS scores. When comparing PPI patterns under placebo versus bupropion, greater negative differences (placebo $>$ bupropion) were associated with lower individual TAS scores. In other words, the higher the individual expression of thrill and adventure seeking, the more subjects were susceptible for the effects of paroxetine and the less susceptible they were to the effects of bupropion (Table 3; Fig. 3). Significant correlations were only evident for PPI measures of the interaction between the NAcc and the avPFC. Parameter estimates of the interaction between the NAcc and raphé nuclei did not correlate with TAS scores.

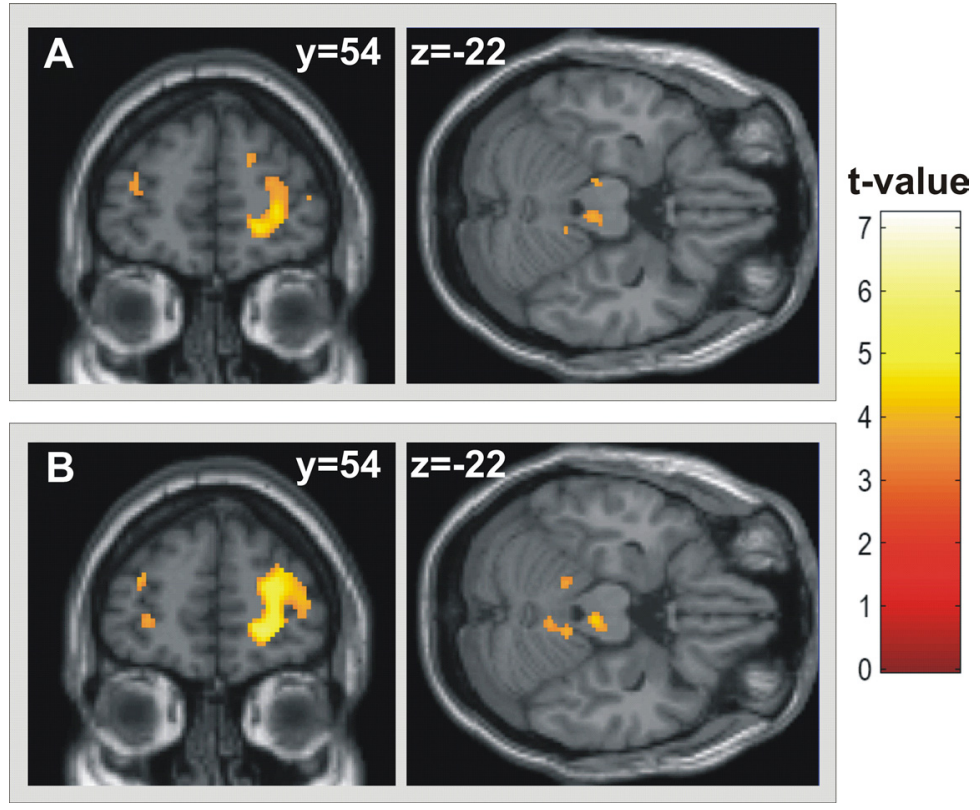

Figure 2. $\quad \boldsymbol{A}, \boldsymbol{B}$, Whole-brain results of psychophysiological interactions of right $(\boldsymbol{A})$ and left $(\boldsymbol{B}) \mathrm{NAcc}$ with the bilateral avPFC and periaqueductal gray in the area of the raphé nuclei significant under paroxetine (threshold of $p<0.001$, uncorrected, minimum cluster size of 10 continuously significant voxels), illustrating the specificity of the region-of-interest results. 
Table 3. Correlations of differential psychophysiological interactions (PPIs) with TAS scores (Thrill and Adventure Seeking subscale of Sensation Seeking Scale, SSS-V)

\begin{tabular}{|c|c|c|c|c|c|c|c|c|}
\hline & \multicolumn{4}{|c|}{$\begin{array}{l}\text { Correlation with degree of differential functional interaction with activ- } \\
\text { ity in left NAcc }\end{array}$} & \multicolumn{4}{|c|}{$\begin{array}{l}\text { Correlation with degree of differential functional interaction with } \\
\text { activity in right NAcc }\end{array}$} \\
\hline & Peak voxel $(x / y / z)$ & Max $z$ value & Peak $r$ & $p$ value & Peak voxel $(x / y / z)$ & Max $z$ value & Peak $r$ & $p$ value \\
\hline \multicolumn{9}{|c|}{$\mathrm{PAR}<\mathrm{PLAC}$ correlation (neg) TAS } \\
\hline avPFC, right & $22 / 58 / 6$ & 3.10 & -0.68 & 0.001 & $22 / 60 / 6$ & 3.28 & -0.71 & 0.001 \\
\hline $\begin{array}{l}\text { avPFC, left } \\
\text { raphe nuclei }\end{array}$ & $\begin{array}{l}-24 / 54 /-4 \\
\text { None }\end{array}$ & 2.87 & -0.64 & 0.002 & $\begin{array}{l}-28 / 48 / 10 \\
\text { none }\end{array}$ & 2.92 & -0.65 & 0.002 \\
\hline \multicolumn{9}{|c|}{ PLAC $<$ BUP correlation (pos) TAS } \\
\hline avPFC, right & $36 / 54 / 8$ & $3.32^{*}$ & 0.71 & $<0.001$ & $22 / 56 / 6$ & 3.03 & 0.67 & 0.001 \\
\hline $\begin{array}{l}\text { avPFC, left } \\
\text { raphe nuclei }\end{array}$ & $\begin{array}{l}-34 / 50 / 8 \\
\text { None }\end{array}$ & $3.64^{*}$ & 0.76 & $<0.001$ & $-32 / 48 / 8$ & 3.23 & 0.70 & 0.001 \\
\hline
\end{tabular}

Threshold at voxel-level: $p<0.01$, uncorrected.

*Results survive FDR correction at $p<0.05$.

$r$, Correlational value; PAR, paroxetine; PLAC, placebo; BUP, bupropion; PAR $<$ PLAC, more negative interaction under paroxetine than under placebo; PLAC $<$ BUP, less negative interaction under bupropion than under placebo; neg, negative; pos, positive.

A

\section{PPI: paroxetine vs. placebo and trait impulsivity}

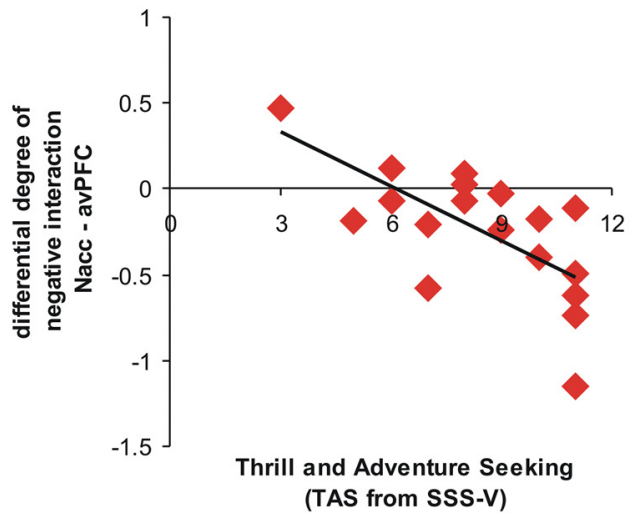

B

PPI: placebo vs. bupropion and trait impulsivity

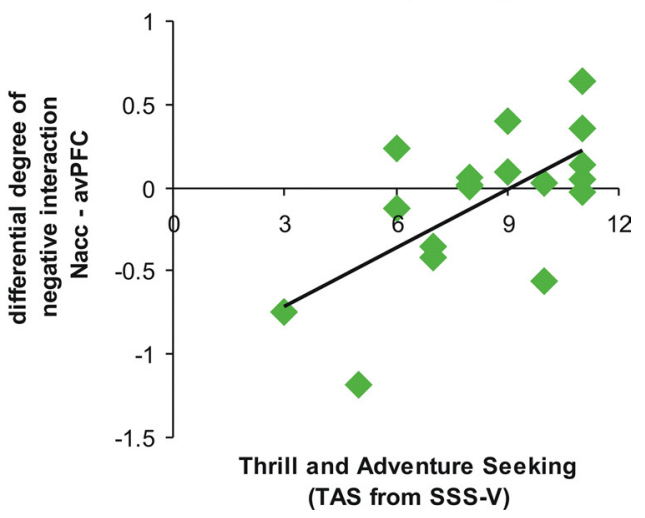

Figure 3. Correlational results of the differential height of negative PPIs between left NAcc and right avPFC with trait impulsivity, as assessed with the TAS subscale of the sensation seeking scale SSS-V. A, More negative PPIs under paroxetine versus placebo are negatively correlated with TAS scores. $\boldsymbol{B}$, Less negative PPIs under bupropion versus placebo are positively correlated with TAS scores.

\section{Discussion}

In the present study, we investigated functional interactions between brain areas that have previously been related to active cognitive control and discounting of secondary rewards (Diekhof and Gruber, 2010). With the difference of using primary rewards and even in the absence of any cognitive control, a significant negative interaction between the NAcc, as part of the mesolimbic dopaminergic reward system, and a region in the avPFC was observed under placebo. These interactions were significantly modulated by two different drugs, the serotonergic antidepressant drug paroxetine and the dopaminergic/noradrenergic drug bupropion. Very similar to the active cognitive control condition of Diekhof and Gruber (2010), treatment with SSRI significantly increased negative coupling estimates, indicating that increased avPFC activities were associated with the observed reduced responsiveness of the NAcc upon processing of primary rewards under this drug. Such a mechanism is in line with an opponency of dopaminergic and serotonergic principles and was further supported by the observation of markedly reduced negative interactions with both regions of interest when subjects had taken bupropion as a dopaminergic drug. Bupropion also led to an increased responsiveness of the NAcc under erotic stimulation, although the difference against placebo was not statistically significant. To further align present results with those of Diekhof and Gruber (2010), we tested whether the observed interactions were related to individual measures of personality. Although with a slightly different realization of the psychological construct of trait impulsivity, we observed that subjects with higher impulsivity scores showed more enhanced negative coupling between the NAcc and avPFC when coupling estimates under the SSRI were compared against placebo. In contrast, this relationship was inverted when comparing coupling estimates under bupropion against placebo, and lower individual TAS scores were associated with higher negative parameters of the estimated interaction.

The observed negative NAcc-avPFC interactions have recently been interpreted as a neural correlate of prefrontal inhibitory control over reward signals in the NAcc (Diekhof and Gruber, 2010). This interpretation is well supported by previous evidence of the existence of projections from orbitofrontal cortices to the NAcc in primates (Haber et al., 1995). Furthermore, the functional interaction of orbitofrontal cortices and mesolimbic dopaminergic brain areas has already been described in experimental animals (Carr and Sesack, 2000; Goto and Grace, 2008). In humans, an exaggerated fMRI signal of the NAcc relative to lateral orbitofrontal activity has been shown in adolescents. The finding was interpreted as the result of a disproportionately earlier maturation of the subcortical reward system relative to inhibitory cortical areas, which might explain increased risk-taking behavior in adolescents and their bias of actions toward immediate over long-term gains (Galvan et al., 2006). Similarly, in obese women, a dysfunctional response of the reward system to food stimuli has been reported along with changed effective connectivities between the NAcc and the orbitofrontal cortex (Stoeckel et al., 2009). Also, previous attempts to target impaired impulse 
control in humans with SSRIs (Hollander et al., 2005) align with the hypothesis of a serotonergically mediated inhibitory control of the prefrontal cortex over reward signals in the NAcc (Duvauchelle et al., 1992).

In accordance with the previous study on NAcc-avPFC interactions and cognitive control (Diekhof and Gruber, 2010), we also observed significant relationships between the personality trait impulsivity and the extent of these functional interactions. Subjects with higher tendency towards risk-taking and sensationseeking behavior appeared more susceptible to medication effects of the serotonergic agent. In contrast, effects of the treatment with bupropion (less negative interactions compared with placebo) were stronger in subjects with lower TAS scores. In the previous study by Diekhof and Gruber (2010), subjects with lower novelty seeking/impulsivity traits expressed a higher negative NAcc-avPFC interaction that in turn was related to a higher inhibitory behavioral control. The authors suggested that the height of NAcc-avPFC functional interaction might be related to a stable disposition for impulsive behaviors, and that less negative functional interactions might be associated with a reduced ability to control desires and impulses. Given this framework, the negative relationship of increasing TAS scores with increasingly negative, paroxetine-modulated NAcc-avPFC interaction estimates, as found in our study, suggests that subjects with a higher propensity for risk-taking behaviors are more susceptible to potentially inhibitory drug effects. Under bupropion, however, the opposite effect emerges: subjects low on risk seeking/trait impulsivity demonstrated more decreased inhibitory NAcc-avPFC interactions, suggesting that a lower propensity for risk-taking behavior promotes the dopaminergic/noradrenergic mechanism of action. While greater risk-taking behavior facilitated the susceptibility to the potentially inhibitory drug effects of paroxetine, less risk-taking facilitated the susceptibility to the disinhibitory effects of bupropion.

An association of bupropion with decreased inhibitory control is in good accordance with previous reports on the detrimental effects of dopamine agonists on impulse control. Increased impulsivity has been observed in dopamine-treated patients with Parkinson's disease or restless legs syndrome (Dodd et al., 2005; Gallagher et al., 2007; Voon et al., 2007; Abler et al., 2009). The dopamine agonistic properties of bupropion may therefore have led to the decreased inhibitory NAcc-avPFC interactions resulting in a less inhibited, increased NAcc activation upon erotic stimulation.

While present results appear to well corroborate previous findings on the interaction between avPFC and NAcc, those previous results were obtained using money as a secondary reinforcer (Diekhof and Gruber, 2010). Therefore it is of note that we could extend those previous results by use of erotic stimuli as a primary reinforcer (Agmo, 2007). Since it is not an absolute condition that neural regulatory mechanisms related to the processing of primary and secondary reinforcers have to be the same, the commensurability of both result patterns independent of specific stimulus characteristics further strengthens the generalizability of the interpretation above.

Parallel to the reciprocal modulation of the NAcc-avPFC interaction by bupropion and paroxetine, psychophysiological interaction analyses also revealed the predicted interactions between the bilateral NAcc and raphé nuclei and their modulation by the different drugs. Treatment with an SSRI enhanced the negative coupling between the NAcc and the key structure of the serotonergic system, and integrates well with previous ideas on a link between SSRI intake and altered processing of primary rewards (Kranz et al., 2010; Boureau and Dayan, 2011).

A limitations to this study is the fact that paroxetine, in addition to its strong affinity for serotonergic receptors, has a weak affinity for muscarinic receptors (Thomas et al., 1987) that may have modulated the results to some extent. Because we only included male subjects, generalizability of the results is limited. Although investigating subjects after $7 \mathrm{~d}$ of medication intake allowed for steady-state conditions, the full spectrum of adaptive changes in neurotransmitter systems that may relate to antidepressant effects could not be captured. The overshoot of serotonergic functioning that is thought to underlie the antidepressant efficacy of SSRIs is only observed after 3-4 weeks (Blier and de Montigny, 1998). Initial inhibitory effects on noradrenergic activity under bupropion recover over a period of $\sim 2$ weeks (El Mansari et al., 2008). According to these findings, statements regarding monoaminergic functioning under paroxetine or bupropion at $7 \mathrm{~d}$ of intake may not cover the full spectrum of the drugs' mode of action. Furthermore, directionality of the influences between investigated brain areas cannot be determined with the method of calculating psychophysiological interactions used.

The present results shed further light on potential mechanisms of how different antidepressants may modulate rewardrelated behavior and associated brain activation. Enhanced negative NAcc-avPFC and NAcc-raphé interactions under SSRIs may help to explain the decreased activity of the ventral striatum under these drugs and the associated deficits in reward-related functions, including well known effects on impulsivity, hedonia, and sexual satisfaction. Understanding of these mechanisms appears to be of high relevance to assure adherence to and efficacy of antidepressive treatment, especially in young individuals. The additional observation on how the existence of a specific and conceptually related personality trait modulated individual drug effects on therapeutically more or less wishful interactions between brain regions may further help in adjusting individualized treatment protocols in the future.

\section{References}

Abler B, Walter H, Erk S, Kammerer H, Spitzer M (2006) Prediction error as a linear function of reward probability is coded in human nucleus accumbens. Neuroimage 31:790-795.

Abler B, Hahlbrock R, Unrath A, Grön G, Kassubek J (2009) At-risk for pathological gambling: imaging neural reward processing under chronic dopamine agonists. Brain 132:2396-2402.

Abler B, Seeringer A, Hartmann A, Grön G, Metzger C, Walter M, Stingl J (2011) Neural correlates of antidepressant-related sexual dysfunction: a placebo-controlled FMRI study on healthy males under subchronic paroxetine and bupropion. Neuropsychopharmacology 36:1837-1847.

Agmo A (2007) Functional and dysfunctional sexual behavior: a synthesis of neuroscience and comparative psychology. London: Elsevier Academic.

Alex KD, Pehek EA (2007) Pharmacologic mechanisms of serotonergic regulation of dopamine neurotransmission. Pharmacol Ther 113:296-320.

Beauducel A, Strobel A, Brocke B (2003) Psychometrische Eigenschaften und Normen einer deutschsprachigen Fassung der Sensation SeekingSkalen, Form V. Diagnostica 49:61-72.

Blier P, de Montigny C (1998) Possible serotonergic mechanisms underlying the antidepressant and anti-obsessive-compulsive disorder responses. Biol Psychiatry 44:313-323.

Boureau YL, Dayan P (2011) Opponency revisited: competition and cooperation between dopamine and serotonin. Neuropsychopharmacology 36:74-97.

Carr DB, Sesack SR (2000) Projections from the rat prefrontal cortex to the ventral tegmental area: target specificity in the synaptic associations with mesoaccumbens and mesocortical neurons. J Neurosci 20:3864-3873.

Clayton AH, Pradko JF, Croft HA, Montano CB, Leadbetter RA, Bolden- 
Watson C, Bass KI, Donahue RM, Jamerson BD, Metz A (2002) Prevalence of sexual dysfunction among newer antidepressants. J Clin Psychiatry 63:357-366.

Diekhof EK, Gruber O (2010) When desire collides with reason: functional interactions between anteroventral prefrontal cortex and nucleus accumbens underlie the human ability to resist impulsive desires. J Neurosci 30:1488-1493.

Dodd ML, Klos KJ, Bower JH, Geda YE, Josephs KA, Ahlskog E (2005) Pathological gambling caused by drugs used to treat Parkinson disease. Arch Neurol 62:1377-1381.

Duvauchelle CL, Levitin M, MacConell LA, Lee LK, Ettenberg A (1992) Opposite effects of prefrontal cortex and nucleus accumbens infusions of flupenthixol on stimulant-induced locomotion and brain stimulation reward. Brain Res 576:104-110.

El Mansari M, Ghanbari R, Janssen S, Blier P (2008) Sustained administration of bupropion alters the neuronal activity of serotonin, norepinephrine but not dopamine neurons in the rat brain. Neuropharmacology 55:1191-1198.

Friston KJ, Buechel C, Fink GR, Morris J, Rolls E, Dolan RJ (1997) Psychophysiological and modulatory interactions in neuroimaging. Neuroimage 6:218-229.

Gallagher DA, O'Sullivan SS, Evans AH, Lees AJ, Schrag A (2007) Pathological gambling in Parkinson's disease: risk factors and differences from dopamine dysregulation: an analysis of published case series. Mov Disord 22:1757-1763.

Galvan A, Hare TA, Parra CE, Penn J, Voss H, Glover G, Casey BJ (2006) Earlier development of the accumbens relative to orbitofrontal cortex might underlie risk-taking behavior in adolescents. J Neurosci 26: 6885-6892.

Goto Y, Grace AA (2008) Limbic and cortical information processing in the nucleus accumbens. Trends Neurosci 31:552-558.

Haber SN, Kunishio K, Mizobuchi M, Lynd-Balta E (1995) The orbital and medial prefrontal circuit through the primate basal ganglia. J Neurosci 15:4851-4867.

Hayes DJ, Greenshaw AJ (2011) 5-HT receptors and reward-related behaviour: a review. Neurosci Biobehav Rev 35:1419-1449.
Hollander E, Sood E, Pallanti S, Baldini-Rossi N, Baker B (2005) Pharmacological treatments of pathological gambling. J Gambl Stud 21:99-110.

Kranz GS, Kasper S, Lanzenberger R (2010) Reward and the serotonergic system. Neuroscience 166:1023-1035.

Labbate LA, Lare SB (2001) Sexual dysfunction in male psychiatric outpatients: validity of the massachusetts general hospital sexual functioning questionnaire. Psychother Psychosom 70:221-225.

Nutt D, Demyttenaere K, Janka Z, Aarre T, Bourin M, Canonico PL, Carrasco JL, Stahl S (2007) The other face of depression, reduced positive affect: the role of catecholamines in causation and cure. J Psychopharmacol 21:461-471.

Price J, Cole V, Goodwin GM (2009) Emotional side-effects of selective serotonin reuptake inhibitors: qualitative study. Br J Psychiatry 195: 211-217.

Reinecke A, Schöps D, Hoyer J (2006) Sexuelle Dysfunktionen bei Patienten einer verhaltenstherapeutischen Hochschulambulanz: Häufigkeit, Erkennen, Behandlung. Verhaltenstherapie 16:166-172.

Seo D, Patrick CJ, Kennealy PJ (2008) Role of serotonin and dopamine system interactions in the neurobiology of impulsive aggression and its comorbidity with other clinical disorders. Aggress Violent Behav 13:383-395

Serretti A, Chiesa A (2009) Treatment-emergent sexual dysfunction related to antidepressants: a meta-analysis. J Clin Psychopharmacol 29:259-266.

Stoeckel LE, Kim J, Weller RE, Cox JE, Cook EW 3rd, Horwitz B (2009) Effective connectivity of a reward network in obese women. Brain Res Bull 79:388-395.

Thomas DR, Nelson DR, Johnson AM (1987) Biochemical effects of the antidepressant paroxetine, a specific 5-hydroxytryptamine uptake inhibitor. Psychopharmacology (Berl) 93:193-200.

Voon V, Thomsen T, Miyasaki JM, de Souza M, Shafro A, Fox SH, DuffCanning S, Lang AE, Zurowski M (2007) Factors associated with dopaminergic drug-related pathological gambling in Parkinson disease. Arch Neurol 64:212-216.

Walter M, Witzel J, Wiebking C, Gubka U, Rotte M, Schiltz K, Bermpohl F, Tempelmann C, Bogerts B, Heinze HJ, Northoff G (2007) Pedophilia is linked to reduced activation in hypothalamus and lateral prefrontal cortex during visual erotic stimulation. Biol Psychiatry 62:698-701. 